\title{
A Review on the Mechanism Between Different Factors and the Occurrence of Autism and ADHD
}

This article was published in the following Dove Press journal: Psychology Research and Behavior Management

\section{Tingting $X_{i}$ \\ Jinlin Wu}

Medical Administration Department, West China Second University Hospital, Sichuan University/West China Women's and Children's Hospital, Chengdu, Sichuan Province, People's Republic of China
Correspondence: Jinlin Wu No. 20, Section 3, South Renmin Road, Chengdu, Sichuan Province, 61004I, People's Republic of China Email I5II833949@qq.com

\begin{abstract}
The incidence of neurodevelopmental disorders especially in children has been on the rise in recent decades, which is possibly caused by many different factors. In order to further explain this situation and arouse enough attention, this review will specifically elaborate genetic aspects and pathogenic hypothesis of these two kinds of neurodevelopmental diseases in children, autism and attention deficit hyperactivity disorder (ADHD) while analyzing the relationship between different environmental toxins and these two disorders. The effects of these pathogenic factors such as heavy metal lead, mercury, polyvinyl chloride (PVC) and polychlorinated biphenyls (PCBs) and their strong pathogenicity will be explained in detail through literature data retrieval and analysis. In addition, other neurotransmitter such as norepinephrine (NE) and serotonin (5-HT) signaling factors coming down to these two neurodevelopmental disorders and how their abnormal concentration present in the blood as well as the completely opposite results among experimental groups and control group will be elaborated. Furthermore, other high-risk and high-exposure factors may contribute to both diseases, such as alcohol and smoking abuse among parents, air pollutants PM2.5 and PM10 in the environment will also be discussed in the review. Since these environmental toxins and other harmful substances discussed in the paper have been linked to an increasing number of children with autism and ADHD in recent decades, from the medical perspective, this review will put forward certain succinct points to the aspects of minimizing relevant exposure or risks in clinical and daily life, as well as feasible suggestions in public health area for children, parents and prospective parents, to curb the growth of these two diseases in part by raising awareness in the population and reducing unnecessary exposure.
\end{abstract}

Keywords: autism, ASD, ADHD, neurotoxins, exposure

\section{Introduction}

In recent years, due to multiple reasons such as the deteriorating environment, the incidence of diseases in human beings, especially in children, is increasing. There are many hypotheses concerning to this hot issue, including changes in the way diseases are diagnosed, vaccines, and even neurotoxic chemicals in the environment. Studies have shown that many neurodevelopmental diseases may be caused by neurotoxic chemicals such as lead, mercury, polyvinyl chloride and PCBs. ${ }^{1}$ From available data, there is quite certain that the incidence of Autism and ADHD has increased over time, and the increase cannot be ignored over the past decades. 
PCB and mercury emissions are strongly associated with Autism in terms of neurotoxin pathogenicity. According to relevant researches, every time mercury or PCB emissions spike, the incidence of Autism seems to increase. At the same time, it is also possible that these neurodevelopmental disorders, particularly Autism and ADHD, are caused by other factors, and it will take years of research to find a direct link. But it is generally agreed that most of the industrial chemicals present in the environment today are known carcinogens, so caution is warranted in public health areas. The public should be careful about the biological accumulation of fish or food contaminated with these chemicals in the body.

\section{Background}

Brain development is a series of evolutionary and degenerative processes regulated by the interaction of cellular and environmental factors. The period of rapid development lasts from the second trimester to the first 24 months of life. These processes are regulated not only by genetics but also by environmental factors. Fetal brain development is particularly sensitive to the environment, prenatal stress can affect neurotransmitters, neuromorphic, neurotrophic factors and cell adhesion molecules and so on, particularly in sensitive stage of acute or chronic regulate cell behavior and gene expression alter neural development, the influence of the distortion of the cell behavior and gene expression form permanent structure remodeling and function program rewoven, lead to brain more susceptible to future stress. Studies have also shown that early malnutrition can affect the hippocampus, an area of the brain associated with spatial learning and memory, and affect its morphological, neurochemical and neurophysiological functions, leading to cognitive learning dysfunction. Prenatal environment factors from various aspects affect fetal brain development, development caused by cerebral ischemia in the phenotype of susceptibility genes, stylized in cellular and molecular level change normal brain cell behavior and specific brain structures, reconstruction of the hypothalamic pituitary adrenal axis, interfere with the key neurotransmitters, change the course of normal brain development, increased neonatal hypoxic ischemic encephalopathy and long-term high susceptibility of neurodevelopmental disorder. ${ }^{2}$

Autism spectrum disorder (ASD) and attention deficit hyperactivity disorder (ADHD) often occur together, and both are highly hereditary while genetic risk variants are hard to find. In terms of whole-gene association analysis, there are some studies that have found specific genetic variations, but very few have found genetic associations that support the excitatory and inhibitory neurotransmitter systems involved in the severity of ADHD and ASD symptoms by analyzing multiple gene variations in a particular genome. For example, glutamate and aminobutyric acid, major excitatory and inhibitory neurotransmitters in the brain, have been shown to be associated with hyperactivity/impulsivity severity, while the GABA genome has been shown to be significantly associated with inhibition. ${ }^{3}$

\section{Autism}

Autism, also called autistic disorder, is a kind of severe nerve widely developmental disorders, which main features are obstacle to verbal and nonverbal communication, social interaction and rigid narrow interest. It usually appears in children before the age of 3 and predisposes to boys. The sex ratio is usually $4: 1 \sim 6: 1$ with quite high morbidity. Autism is a disorder that affects children's social skills. Widespread autism is known as autism spectrum disorder (ASD). It is estimated that 3.4 out of every 1,000 children aged 3-10 have ASD. ${ }^{4}$ The incidence has been on the rise in recent years, causing serious damage to children's physical and mental health, doing harm to the families and society, which has aroused extensive attention from all over the world. However, in terms of factors influencing the etiology of ASD, Ma et $\mathrm{al}^{5}$ believed that good family upbringing was the protective factor.

\section{Genetic Aspects and Pathogenic Hypothesis of ASD} The role of genetic factors in the development of autism has been demonstrated in earlier twin studies: the prevalence of autism in the monozygotic twins is significantly higher than in the dizygotic twins. Studies have confirmed the occurrence of autism some gene variations may be due to the influence caused by the biological signaling pathways related to brain development. One of the more recognized signal pathway theory is about RAS pathway. RAS is a small guanine binding protein, which plays an important role in the growth and development of eukaryotic cells. Some genes on the chromosomes of autistic children have deletion, duplication and other common mutations, and these locations contain important genes and enzyme cutting sites that affect RAS pathway, thus causing initiation effects in the regulation and expression. Because of the role synapses play in brain development, it has long been suspected that synaptic dysfunction may be the cause of autism, and genetic mutation analysis has found many mutations in genes along the way that may be linked to Autism. ${ }^{6}$ According to Tan et al, ${ }^{7}$ although multiple gene loci and susceptibility genes have been reported successively, 
genetic studies can only explain $20 \% \sim 30 \%$ of heritability. So far, most of the etiological studies on childhood Autism support that Autism is a neuropsychiatric developmental syndrome caused by external environmental factors affecting individuals with genetic susceptibility to it. Meanwhile, social and emotional disorders may be mainly related to dysfunction of the limbic system which is the medial temporal lobe of the brain. ${ }^{8}$ Vaccarino et $\mathrm{al}^{9}$ also pointed out that RAS pathways may explain some symptoms of Autism, such as macrocephaly happened in about $20 \%$ of patients. This symptom is caused by the rapid development of neural cells in the embryonic brain. It may be caused by the disturbance of the fibroblast growth factor (FGF) signal regulation mechanism or the proliferation of neural stem cells before birth. Adviento et $\mathrm{al}^{10}$ also illustrated that certain complications of Autism, such as fragile $\mathrm{X}$ syndrome and tuberous sclerosis (TS), have certain mutant proteins linked to the RAS pathway.

Though abnormalities in brain structure and function are widely believed to be linked to neurodevelopmental disorders, the exact cause of Autism is still unknown. Most scientists believe the deformity is the result of a combination of genetic and environmental factors. Other theories that have been investigated include the link between vaccines, problems during pregnancy, and genetics. ${ }^{11}$ Autism has long been considered primarily a genetic disorder with a number of studies support this view. However, in recent decades, scholars have found through in-depth and extensive research that Autism is a biological disease with multiple causes, mechanisms and phases while environmental factors are closely related to it.

In terms of neuroimaging, positron emission tomography (PET), functional magnetic resonance imaging (fMRI) and single photon emission computer tomography (SPECT) were used to find structural and metabolic abnormalities in the limbic system, brainstem, cerebellum and related cortex. In neuropathology, through animal model studies, some researchers have found that the limbic system of the brain may be closely related to Autism. The limbic system is primarily associated with Autism in the amygdala and hippocampus, an almond-shaped subcortical center that controls human emotion and aggression. Social withdrawal, compulsive behavior, ignorance of dangerous situations, inability to extract information from memory stores, and inability to adjust oneself to new events or circumstances are associated with damage to the hippocampus. The amygdala is also responsible for responses to a variety of sensations. Individuals with Autism have been found to have trouble responding to these stimuli, and surgical damage or removal of the amygdala can reproduce the aggressive and emotionally detached behavior of autistic children in animals. Hippocampus is a center for learning and memory. The inability of children with Autism to use previously stored information in new situations may be linked to this part of the brain. It is also believed that ASD is related to heredity or genetic abnormality rather than one or a few genes. Cytogenetic studies have also found that people with specific chromosomal aberrations have a higher incidence of getting Autism. At present, except for no specific Autism susceptibility genes found on chromosome 14 and chromosome 20, other chromosomal abnormalities are all considered to be correlated with the incidence of Autism. For example, 7q31-q35, 15q11q13 and sex chromosomes are widely investigated in this area. Researchers found that $1 \%$ to $3 \%$ of people with Autism have abnormalities in chromosome 15 , mostly gotten from their mothers. There is now a consensus that Autism, or core pedigree, is related to mutations on chromosomes 7 and $15 .^{12}$ Similar conclusion has been found by Feng et $\mathrm{al}^{13}$ and $\mathrm{Li}$ et al, ${ }^{14}$ who studied 42 cases of patients and found relevant auxiliary examination of SPECT abnormality rate was $93 \%$. The prompt SPECT examination result has a certain value to the diagnosis of Autism, which mainly reduced the left side of the brain blood flow perfusion, frontal lobe on the temporal lobe and the limbic system (hippocampal gyrus cingulate insula, etc.). The exception results of these parts may lead to social communication disorders in children with language communication barriers, stereotyped behavior and feeling or movement disorders.

In the field of neuroelectrophysiological testing, Kong \& Duan ${ }^{15}$ selected clinically children who were diagnosed with Autism from 2001 to 2007. They studied 30 cases of BAEP and EEG examination data analysis results and found: BAEP normal 11 cases (37\%), 19 cases (63\%); EEG normal 23 cases (77\%), 7 cases $(23 \%)$. The results show that although the abnormal rate of BAEP is different from that of EEG (the former is obviously higher than the latter), it is indisputable that some autistic children have abnormal BAEP and EEG, and the abnormality of BAEP may be related to the characteristics of Autism itself. Duan et $\mathrm{al}^{16}$ found some possible gut microbes associated with Autism or flora composition. Since most gut microbes cannot by in vitro culture, the potential reason of causing Autism may be microbe in vitro culture of bacteria. Small amounts of them can attach to the lining of the intestine, be distributed in the small intestine, or be present in the feces. Under the condition of current technology, it is impossible to complete sampling and analysis of them, so this speculation is still in the stage of theoretical research. 


\section{ADHD}

Attention Deficit Hyperactivity Disorder (ADHD) is a common neurobehavioral disorder that affects children and persists through adolescence and adulthood. ${ }^{17}$ This type of disorder makes it difficult for children to concentrate and focus on one single task. A large number of studies have confirmed that $70 \%$ of ADHD symptoms can last to adolescence while $30 \%$ of them may last until adulthood in recent years. ADHD patients generally suffer from a variety of mental disorders with increased risks of anti-social behaviors and crimes related to substance dependence in later stages of their lives. Therefore, ADHD has been regarded as a chronic lifelong disease which has become an important public health problem. ${ }^{18}$

\section{Genetic Aspects and Pathogenic Hypothesis of ADHD}

Neurodevelopmental and developmental factors can also trigger children of getting ADHD. The condition is usually diagnosed in preschoolers and early elementary schoolers, which provides a structured environment that allows symptoms to become apparent. It has been observed that the structure and function of different parts of the brain of ADHD children are different. Although there is no known cause, problems during pregnancy or environmental toxins may affect children's brain development. ${ }^{19}$

The exact cause of ADHD is still unknown, but it is widely acknowledged that there is a strong neurobiological basis. Changes in brain structure are thought to be the main cause of the disease. Brain scans showed that the brains of children with ADHD were $4 \%$ smaller than those without the disorder. Neurotransmitters, which are called brain's chemical messengers, perform poorly and found with less activity in areas of the brain responsible for activity and attention in ADHD patients. ${ }^{20}$ Recent data suggest that ADHD has neurophysiological basis abnormality, and hyperactivity and inattention may be related to insufficient function of catecholamine system in the brain. Some scholars examined hyperactivity children and found that local blood flow in the prefrontal cortex of them was hypoperfusion. Brain imaging also showed cortical atrophy in young adults with a history of ADHD in children. Recent PET studies have also discovered that the density of dopamine receptors is associated with childhood development, leading to specific changes in dopamine receptor density which do not occur until adolescence. The area most susceptible to hyperactivity in children is thought to be the dopa pathway in the anterior lobe. ${ }^{21}$ ADHD in children can be understood as a comprehensive result of the dysfunction of information transmission between neurons in the brain and the disorder of the mutual restriction between inhibitory synapses and excitatory synapses. Wang ${ }^{22}$ thought that abnormal metabolism of neurotransmitters in the brain may be caused by insufficient quantity of neurotransmitters, leading to the consequence that the information cannot be delivered in time.

Another possible source is exposure to some toxins during pregnancy of their mothers. The brain begins to develop from conception to the first year of life. Alcohol and cigarettes distort the growth of nerve cells. Children with fetal alcohol syndrome have the same symptoms of inattention, hyperactivity and impulsivity as children with ADHD. Other drugs, such as cocaine, can affect the normal development of brain receptors, which may also lead to the disorder. Lastly, toxins and everyday activities in the environment may be linked to disruption of brain development and regular processes. ${ }^{23}$

\section{Methodology Purpose of Study}

The purpose of this review is to find a series of risk factors that may lead to Autism and ADHD in children from a certain amount of professional literature. At the same time, some possible pathogenic factors in neurobiological area were also discussed in order to attract more attention from the public. In addition, in public health area, especially in early screening process of children with these neurodevelopmental disorders, it is hoped to make a small contribution to providing them with early intervention and proper treatment.

\section{Research Method}

The main research method of this review is literature reading, through reading a large and sufficient amount of literature, summarize valid data and conclusions or relevant hypothesis, determine the pathogenic mechanism of certain specific factors may lead to autism and ADHD; looking for methods to reduce the risk of exposure to diseases of newborns; making contributions to the public health care of women and children.

\section{Data Collection and Analysis}

Literature searched in PubMed, Knowledge Network, CQVIP and other databases; keywords including "Autism", "ADHD”, "neurotoxin", "environmental toxin", "exposure", etc. From official sources such as the Centers for Disease Control in different regions, the National Institutes of Health, and the Environmental Protection Agency, papers and 
articles, tabulating relevant data and analyzing the causes of the increased incidence of children in recent years mainly from the aspect of pathogenic mechanism.

\section{Main Findings}

In general, both autism (ASD) and attention deficit hyperactivity disorder (ADHD) are polygenic and heterogeneous diseases, which explains the difficulty of identifying underlying genetic factors. ${ }^{3}$ According to Bedrosian et al, ${ }^{1}$ to find out whether there was a link between environmental toxins and neurodevelopmental disorders, an analysis of large amounts of data were collected between 1990 and 2007, including toxins such as lead, mercury, PCBs and PVC. The research found that only two neurobehavioral disorders, which are autism and ADHD, had rather clear information of their prevalence over the years.

Neurotoxin is any compound derived from an exogenous source (ie, the environment) that affects neurodevelopment. The major toxins include mercury, lead, PVC (polyvinyl chloride) and PCBs (polychlorinated biphenyls). Exposure to harmful toxins above the threshold or in large doses during the early stages of pregnancy or childhood (especially during infancy) could lead to many diseases, especially neurodevelopmental diseases, fertility or even death. ${ }^{24}$ Since humans may be exposed to lead, mercury, PVC, and PCBs through inhalation, ingestion, or merely through skin contact like absorption, and they are used to produce many everyday products, which poses a bigger risk to the whole population and the environment. One of the serious results is that the number of children getting autism and ADHD is increasing over the course of decades.

\section{Discussion}

\section{Different Neurotoxins Contribute to Autism and ADHD}

Data collected from a rather accurate survey shown in Figure $1^{1}$ of long-term data in 10 different US states suggest and provide a possible link between environmental toxins such as lead, mercury, PCBs, and autism rates; as well as a graph of the percentage of children diagnosed with ADHD. As can be seen from the statistical data in Table $1,{ }^{1}$ the incidence of autism and ADHD in the United States from 1992 to 2003 was accompanied by an increase in the content of toxic substances such as heavy metals in the environment, and the number of children with the disease also showed a general trend of increasing year by year.

\section{Lead and Mercury}

Lead is involved in certain serious diseases such as childhood leukemia. At the same time, lead has also been proved by quite amount of studies of its link to many teratogenic or defective diseases. Nowadays children are still at risk for lead exposure, especially for those who live in houses with lead-based paint. According to National Safety Council, ${ }^{25}$ it is estimated that more than 400,000 children under the age of six have high levels of lead in their blood. The highest risk groups are fetuses and younger children groups for their fastest development in body. Exposure to low threshold concentrations of lead in children under 6 years of age can lead to reduced IQ, learning disabilities, attention deficit disorders, behavioral problems, developmental delays and hearing impairment. High levels of exposure can cause a range of more severe reactions such as mental retardation, coma, and even death. Not only does lead interfere with brain and neural development in children, it also affects blood formation and kidney function. During pregnancy, lead can be transferred from the mother through the "blood-placental barrier" to the developing fetus. In addition, studies have suggested that the stress of pregnancy can cause lead to be released from a mother's bones into her bloodstream and eventually into the fetus, leading to toxic effects and even birth defects. Results of a clinical control study ${ }^{26}$ showed that there were statistically significant differences in perinatal lead poisoning, toxic exposure, partial diet and breastfeeding between the two tested groups $(\mathrm{P}<0.01)$.

Mercury is widely found in nature and such as air, water and soil because it is a natural element on its own. Meanwhile, even small amounts of mercury can cause serious health problems. Very limited amount of mercury exposure during pregnancy can pose a threat to the development of embryos and fetuses in the womb early in life, especially do harm to the development of one's nervous system. Similar to lead, mercury exposure remains the highest risk in children under 6 years of age and fetuses. Other relevant factors include the chemical form of mercury during the time of exposure, the route of exposure, and the current physical condition of the subjects. ${ }^{1}$ Historically, there have been outbreaks of methylmercury in areas where fish are often consumed in large quantities. Mothers with no signs of neurodevelopmental damage have given birth to severely disabled babies, which directly suggested that the nervous systems of fetuses are 
$\%$ Of Children $<6$ Years Old with

Blood Lead Levels $>10 \mu \mathrm{g} / \mathrm{dL}$

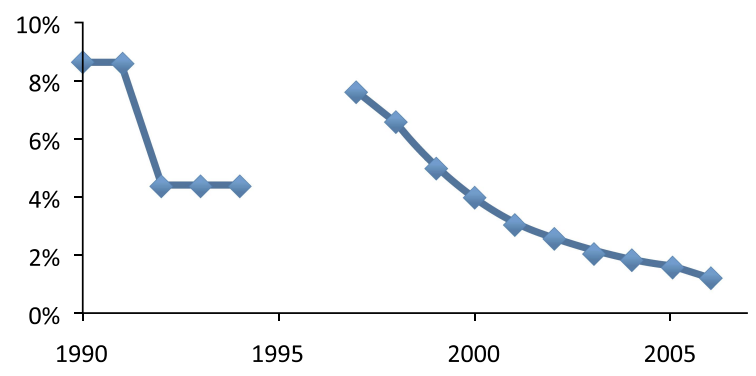

\% of Children 6-22 with Autism

Mercury Emissions
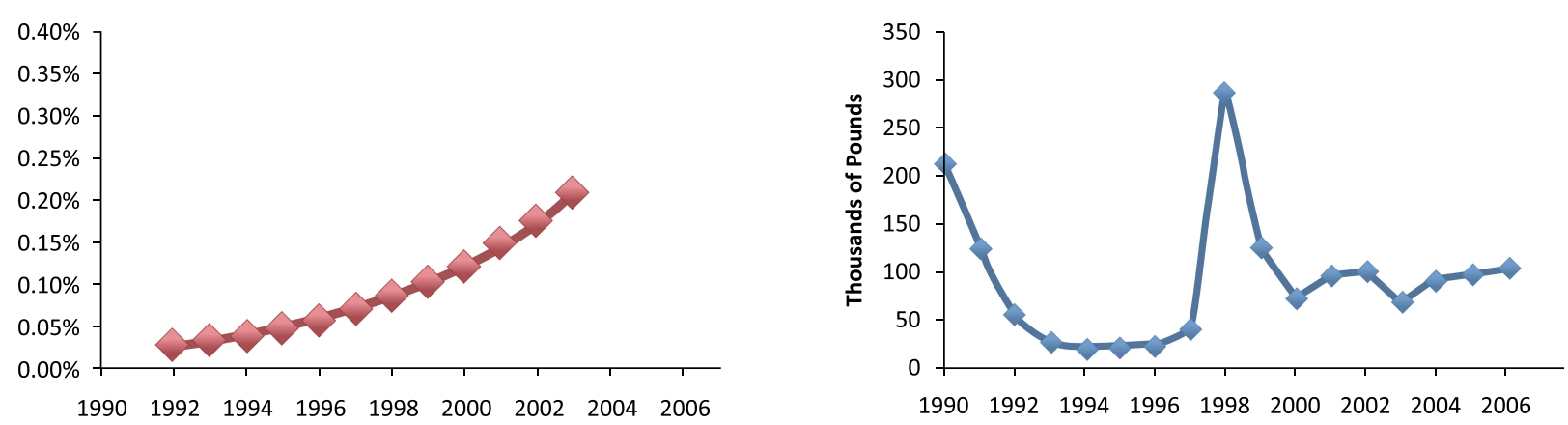

$\%$ of Children Diagnosed with ADHD
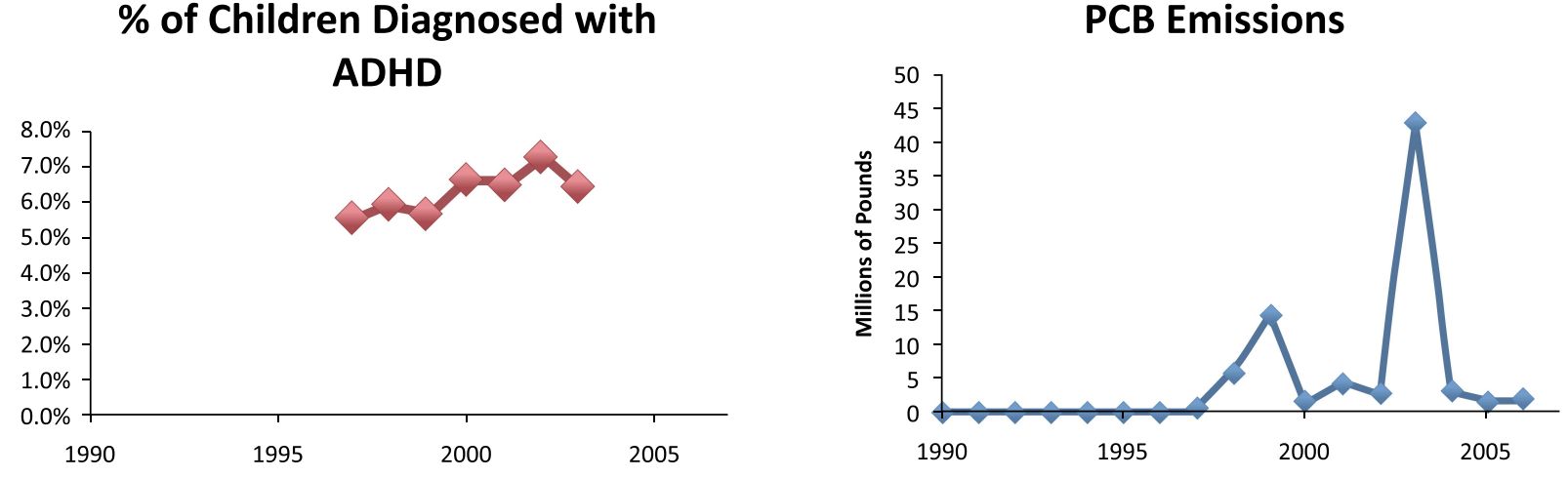

PVC Consumption

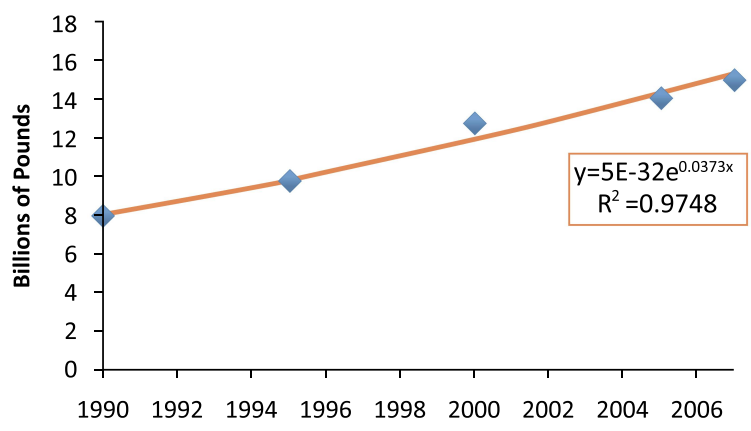

Figure I Comprehensive diagram of prevalence of Autism \& ADHD and the amount of different kinds of neurotoxins in the United States between 1990 and 2007. Reproduced from Smith EE, Charest JM, Bedrosian KR, DeVault VL Neurotoxic chemicals in the environment. 2008.' 
Table I Comparison of Trends in Neurodevelopmental Disorders and Neurotoxins in the General Environment in the United States Between 1992 and $2003^{\prime}$

\begin{tabular}{|c|c|c|c|c|c|c|c|}
\hline \multicolumn{8}{|c|}{ US Totals } \\
\hline Year & Mercury & Lead & РСB & PVC & ADHD & Autism & $\begin{array}{l}\text { Autism\% } \\
\text { Age 6-22 }\end{array}$ \\
\hline 1992 & 55998 & $4.40 \%$ & 427320 & & & 15292 & $0.0257 \%$ \\
\hline 1993 & 26916 & $4.40 \%$ & 164470 & & & 18695 & $0.0311 \%$ \\
\hline 1994 & 20952 & $4.40 \%$ & 94962 & & & 22329 & $0.0368 \%$ \\
\hline 1995 & 23912 & & 34432 & 9.83 & & 28720 & $0.0469 \%$ \\
\hline 1996 & 25042 & & 97945 & & & 33999 & $0.0549 \%$ \\
\hline 1997 & 38507 & $7.61 \%$ & 987640 & & $5.5 \%$ & 42126 & $0.0674 \%$ \\
\hline 1998 & 289518 & $6.50 \%$ & 6127249 & & $5.9 \%$ & 53644 & $0.0850 \%$ \\
\hline 1999 & 126792 & $5.03 \%$ & 14598073 & & $5.6 \%$ & 65607 & $0.1030 \%$ \\
\hline 2000 & 71884 & $3.96 \%$ & 1722883 & 12.85 & $6.6 \%$ & 78928 & $0.1197 \%$ \\
\hline 2001 & 95690 & $3.03 \%$ & 4646321 & & $6.4 \%$ & 97994 & $0.1469 \%$ \\
\hline 2002 & $10004 \mid$ & $2.56 \%$ & 3155954 & & $7.2 \%$ & II807| & $0.1752 \%$ \\
\hline 2003 & 68231 & $2.00 \%$ & 43228331 & & $6.4 \%$ & 140254 & $0.2061 \%$ \\
\hline
\end{tabular}

more susceptible to toxic mercury compounds than those of adults. ${ }^{27}$

\section{PCBs (Polychlorinated Biphenyls) and PVC (Polyvinyl Chloride)}

PCBs are not only carcinogens, but also neurotoxins that have been shown to cause many neurodevelopmental diseases. Polychlorinated biphenyls (PCBs), due to their extreme stability, can be naturally decomposed in natural environments for many years. These toxins are stored in human and animal adipose tissue and tend to bind to sediments. They can be combined with seafloor sediments through a process of bioaccumulation and consumed by organisms, birds, fishes and human. Some clinical case reports have shown that pregnant women with high levels of PCB in their blood are more likely to give birth to offspring with a variety of neurodevelopmental disorders, such as lower IQ and lower birth weight. PCBs are wellestablished neurotoxins, so environmental PCBs may also be associated with increased rates of neurodegenerative diseases such as childhood autism, cerebral palsy, and muscular dystrophy. ${ }^{1}$ When exposed to such an environment, the development of the infants' brain can be severely impaired.
Today, PVC is widely used in the manufacture of rubber shoes, raincoats and other goods, but there have been many studies on its harmful effects in recent years. When PVC is burned, buried, or even used, it releases toxic substances into the atmosphere. PVC production process is designed with the use of chlorine and produce two harmful substances vinyl chloride, a dangerous carcinogen, as well as ethylene dichloride. Both of them can do harm to the kidney, lung, cardiovascular, immune and nervous systems. Phthalates and bisphenol A were added as softeners while these chemicals are directly or indirectly released to the environment, causing harmful effects to human and possibly lead to liver damage, damage to the reproductive and nervous systems or even cancer. Children are most often exposed to PVC through sucking or skin contact with plastic toys. Plastic baby bottles and cutlery used by infants are also an important way of exposure. ${ }^{1}$

\section{Monoamine Neurotransmitters in Blood Contribute to Autism and ADHD}

For the pathogenesis of children with Autism and ADHD, it is worth noting that blood monoamine neurotransmitter in the central nervous system is involved in many physiological reactions. Norepinephrine and Serotonin in the brain 
exist two kinds of function of the phase antagonism mature neurotransmitter. They play important roles in the regulation of mental activity, emotional response and other behavioral patterns. They are important factors in the development of Autism and ADHD in children as well. From a neurobiochemical point of view, there were some article data show that: children with Autism and ADHD have shown changes in NE and 5-HT in their blood. ${ }^{28}$ Generally, NE and 5-HT are in a balanced state of interaction to maintain the appropriate behavior spectrum. Once this balance disturbed, behavior disorders are likely to occur.

\section{Monoamine Neurotransmitters in Blood-Norepinephrine (NE)}

According to Luo et $\mathrm{al}^{28}$ since the level of NE in the blood reflects the excitability of the sympathetic nerve, the level of $\mathrm{NE}$ in the blood of children with ADHD is significantly higher than that of normal ones, which may be one of the causes of hyperactivity in children. As shown in Table 2, children with Autism showed the opposite trend (NE levels were significantly lower than those in normal children), reflecting different disorders of arousal and inhibition.

\section{Monoamine Neurotransmitters in Blood-Serotonin (5-HT)}

Luo et $\mathrm{al}^{28}$ also pointed out that normally serotonin inhibits and stabilizes higher brain regions and mental activity. Children diagnosed with ADHD whose 5-HT content in blood was significantly lower than the normal ones (also shown in Table 2). Probably leading to hyperactivity, inattention and impulsivity. One of the reasons for children with Autism is that 5-HT in their blood is significantly higher than that of normal ones. It may be one of the reasons for autistic children's withdrawal behavior, communication difficulties and communication difficulties. At the same time, it can be concluded from the data in Table 2 that, compared with the data in the Control group, ie, the normal children group, children in the autism group and

Table 2 Comparison of Monoamine Neurotransmitters NE and $5-\mathrm{Ht}$ in Blood of ADHD and Autism Children with Normal Infants $(\bar{x} \pm \mathrm{s})$

\begin{tabular}{|l|l|l|l|}
\hline Group & $\mathbf{n}$ & NE $(\boldsymbol{\theta} \mathbf{g} / \mathbf{m L})$ & $\mathbf{5}-\mathbf{H T}(\mathbf{n g} / \mathbf{m L})$ \\
\hline ADHD group & 35 & $687 \pm 124^{*}$ & $1.0 \pm 0.3^{*}$ \\
\hline Autism group & 35 & $176 \pm 14^{*}$ & $16.3 \pm 8.6^{*}$ \\
\hline Control group & 40 & $359 \pm 84$ & $4.7 \pm 1.2$ \\
\hline
\end{tabular}

Note: Compared with the control group, ${ }^{*} p<0.01$. the ADHD group showed an opposite trend in the proportion of specific neurotransmitters in the comparative experiment of the data difference and effectiveness.

\section{Other Factors Contribute to Autism and ADHD \\ Manganese and Polycyclic Aromatic Hydrocarbons (PAHs)}

Manganese is a natural trace element in soil, water and plants. Manganese metal is mainly used in steel production to improve hardness, stiffness and strength. Several studies have linked excessive manganese exposure to neurodevelopmental diseases in children. According to Zhang and Yan, ${ }^{29}$ most of manganese in the brain stays in the form of pancreatic phthalein amine synthetase, which is the excitatory neurotransmitter pancreatic phthalein amine metabolization key enzyme. This enzyme is important for nerve excitation, inhibition and balance. But excess amount of manganese can alter this balance, causing dopamine metabolism disorders in the nigra striatum after absorption into human (NPC) cells, leading to neurologic symptoms of manganese toxicity. This could be associated with lower math scores, lower psychological functioning and ADHD.

Polycyclic aromatic hydrocarbons (PAHs) are widely found in urban air pollutants, mainly from the combustion of fossil fuels and other organic materials, which are commonly considered to be neurotoxic. One study suggests that exposure to PAHs in the air in New York City may be linked to behavioral problems in ADHD children. In the nine-year study, conducted in New York City, researchers determined the association between behavioral problems with ADHD and prenatal PAHs exposure by following the children of African-American and Dominican women in New York City over time, and finally conducted a professional evaluation of postpartum exposure. The results suggested that high prenatal exposure to PAHs may increase the risk of ADHD behavioral problems in the fetus after birth. However, the mechanisms by which PAHs exposure may affect brain development are not fully proven yet. Endocrine disruption, reduced exchange of oxygen and nutrients due to placental growth factor receptors, DNA damage, and epigenetic changes are several suspected pathways. In addition, since brain development and growth normally continue throughout the whole childhood, direct exposure to them after birth may also affect neurodevelopment and behavior in children. ${ }^{30}$ 


\section{Other Associated Factors}

In a study ${ }^{21}$ of 28 children with ADHD, four of them suggested that mild brain impairment in some children may be related to genetic and environmental factors. In addition, recent studies have found that the incidence of some mental diseases such as alcoholism and pathological personality among parents is higher than that of the control group. All suggested that the clinical symptom fluctuation of hyperactivity children was sometimes related to different places and activities. The phenomenon that their symptoms fluctuate with the scene indicates that the changes of hyperactivity children's symptoms are affected by the environment as well. In addition, the incidence of brain trauma, asphyxia and parental dissonance were statistically different between the two groups $(\mathrm{P}<0.05)$.

Moreover, it is also suggested by Huang and $\mathrm{Jin}^{31}$ that the occurrence of ADHD is associated with genetic factors, perinatal conditions, family psychological factors, nutrition and dietary factors. Air pollutants, especially PM2.5 and PM10, may increase the risk of developing Autism, but there may exist bias in the results of epidemiological studies. According to $\mathrm{Ma}$ and $\mathrm{Li}^{2}$, exposure of tobacco smoke before and during pregnancy, including smoking and passive smoking by pregnant women, has a number of negative effects on the fetus, such as birth defects, preterm delivery, intrauterine growth restriction, childhood obesity, neurodevelopmental development and behavioral abnormalities. The toxic side effects of tobacco smoke may be caused by chronic hypoxia in the fetus. Through the direct effect of nicotine or smoking caused by poor appetite and food consumption leading to malnutrition, may also increase placental resistance; reduce uterine blood flow; increase carbon and oxygen hemoglobin; which could all contribute to the fetal intrauterine chronic hypoxia environment. The mechanism of fetal brain dysplasia induced by nicotine exposure is considered to be caused by stimulation of acetylcholine release. Passive smoking during pregnancy, like active smoking, will increase the risk of fetal neural development and behavioral abnormalities. Children exposed to tobacco smoke will have a 2.5 times higher risk of getting ADHD than children whose mothers are not exposed to tobacco during or before pregnancy.

\section{Conclusion and Recommendations Conclusion}

To sum up, there may exist a possible link between the harmful effects of certain environmental toxins and neurotoxins in the pathogenesis of neurodevelopmental disorders, in this review, autism and ADHD. The number of children with these two disorders has soared over the past few decades, linking to early and excessive exposure to different kinds of environmental toxins such as lead, mercury, PCBs and PVC. It is worth attention that children of age 6 or younger with blood lead levels higher than $10 \mu \mathrm{g} / \mathrm{dL}$ are more susceptible to environmental toxins during early development, and they are more profound effects since blood lead levels are closely related to one's direct exposure to lead.

In the meantime, factors like monoamine neurotransmitter shall also be considered in the process and mechanism of neural signaling since they are rather closely related to children's behavior and the detection of norepinephrine and serotonin may play a role in promoting the understanding of children's behavior patterns. Moreover, other associated factors such as manganese, PAHs, alcoholism, active and passive smoking, parents' mental health, perinatal conditions, family psychological factors, nutrition and diet factors, air pollutants, especially PM2.5 and PM10 could all have an effect on children getting these two diseases, while the specific pathogenic mechanism still needs further research.

\section{Prevention and Intervention}

According to Harris and Blain, ${ }^{32}$ the potential health risks of exposure to toxic environmental agents are now a major public health issue and it is increasingly important to develop expertise and facilities to investigate neurotoxicity in humans. Special attention shall be paid to two priority areas, one of which is the impact of long-term exposure to low levels of environmental neurotoxins on developing fetuses and growing infants. At the same time, early detection, early treatment, early intervention and professional guidance are particularly important in the treatment of these two diseases. In public health areas, it is particularly crucial to carry out early publicity and education work due to the differences in education and reception level among citizens. Some parents may ignore or be reluctant to acknowledge the symptoms their child shows in the early stages of the illnesses, making it more difficult to diagnose and treat under these circumstances. Therefore, it cannot be neglected in future work such as carry out targeted census in communities. The guidance of effective psychological evaluation should be also be advocated. At the same time, strengthening the health care during pregnancy, especially in the perinatal period and early stages of 
pregnancy; paying attention to avoid infection; using antibiotics with caution; carrying out regular prenatal examinations are some intervention measures worth advocating. 33

\section{Reduce Exposures}

Since potential neurotoxin can be absorbed by inhalation, skin contact, food intake and other contacts then stored in human tissues, direct contact and skin absorption pathways are significantly more likely to produce clinical symptoms. But direct application to nerve tissues is less commonly found in real cases. Generally, most exposure pathways are through inhalation, intake of food and water, or absorption by skin. The process of entering the human circulatory system from these sources is highly variable, and unless appropriate biomarkers are available to assess the body's burden of toxins, it is extremely unscientific to calculate the dose of toxins intuitively. Therefore, it is particularly important to reduce exposure factors when quantification is impossible. $^{32}$ In simple terms, it is impossible to avoid all environmental toxins, but it is crucially important to limit your own exposures. People who need to pay special attention to are the pregnant mothers. Reducing the potential possibility of adverse effects on the fetus to the minimum or eliminating risk exposure during pregnancy, especially during the first trimester is extremely crucial as the development of the nervous system plays an important role in embryonic development.

\section{Other Preventive Measures}

Regular testing of children's blood lead. Once increased, use calcium supplement, pectin or other effective methods under the guidance of doctors to drive lead out of the body. It is also suggested for these children to consume certain amount of lea-removal-effect food, such as kelp and beans. ${ }^{34}$ Liu $^{35}$ thought that the state of existing organizations like Autism Rehabilitation Resources at present cannot meet the needs of children diagnosed with the disease. It is urgently needed to provide more of them with effective rehabilitation, which to a large extent, depends on the active participation of their parents. The parents should regularly pay visits to the Autism Rehabilitation Facilities for family therapy consultation and evaluation, in order to ensure the quality and effects of the treatment.

\section{Disclosure}

The authors report no conflicts of interest in this work.

\section{References}

1. Smith EE, Charest JM, Bedrosian KR, DeVault VL Neurotoxic chemicals in the environment. 2008.

2. Ma N, Li L. Effects of fetal environmental factors on brain development. Chin J Child Health. 2015;23(8):821-823.

3. Naaijen J, Bralten J, Poelmans G, et al. Glutamatergic and GABAergic gene sets in attention-deficit/hyperactivity disorder: association to overlapping traits in ADHD and autism. Transl Psychiatry. 2017;7(1):999. doi:10.1038/tp.2016.273

4. National Institutes of Health. What are the autism spectrum disorders? National Institutes of Health; 2008.

5. Ma J, Kuang G, Heng Z, Zhao Y, Ma A, Han X. Analysis of etiology and influencing factors of autism spectrum disorders. Chin J Child Health Care. 2015;23(6):647-649.

6. Cao T, Yu D, Huang F. Role of genetic and environmental factors in autism. Chin J Clin. 2015;9(21):93-97.

7. Tan J, Gao X, Su L. Progress in etiology of childhood autism. Chin $J$ Pediatr. 2013;28(2):143-146.

8. Li N. New advances in neural mechanisms of autism. Psychol Sci. 2001;24(2):249-250.

9. Vaccarino FM, Grigorenko EL, Smith KM, Stevens HE. Regulation of cerebral cortical size and neuron number by fibroblast growth factors: implications for autism. J Autism Dev Disorders. 2009;39 (3):511-520. doi:10.1007/s10803-008-0653-8

10. Adviento B, Corbin IL, Widjaja F. Autism traits in the RASopathies. $J$ Med Genet. 2014;51(1):10-20. doi:10.1136/jmedgenet-2013101951

11. Autism Society of America. Improving the lives of all affected by autism. About Autism. 2008.

12. Deng M, Lao S. New progress in clinical research of autism spectrum disorders (DSM-5 New Standard). Chin $J$ Health Psychol. 2016;4:481-490.

13. Feng S, Zhang J, Zhu M, Zou X, Tang C. Analysis of 213 cases of autism in children. Chin J Child Health Care. 2003;11(2):84-86.

14. Li C, Zhai J, Yang Y. Etiological research and treatment of childhood autism. Shandong J Psychiatry. 2006;19(4):303-307.

15. Kong F, Duan L. Clinical and neuroelectrophysiological detection of autism in children. $J$ Clin Neuroelectrophysiol. 2008;5:301-303.

16. Duan Y, Wu X, Jin F. Research progress on etiology and treatment of autism. Sci Chin. 2015;45(9):820-844.

17. Centers for Disease Control and Prevention. What is attention-deficit/ hyperactivity disorder (ADHD). Department of Health and Human Services; 2005.

18. Wang A. Risk factors and etiology of attention deficit hyperactivity disorder. Chinese Commun Phys. 2010;30:3.

19. National Institute of Mental Health. Attention deficit hyperactivity disorder. 2008.

20. Mayo Clinic Staff. Tools for healthier lives: mayoclinic.com; attention-deficit/hyperactivity disorder (ADHD). 2007.

21. Xiao C. Etiology of 28 cases of hyperactivity disorder in children. Chin J Physicians. 2001:S1.

22. Wang X. The Cause and Pathogenesis of ADHD [doctoral dissertation]. 2006.

23. Jaffe J, Benedictis TD, Segal R. Possible causes and risk factors of ADHD. 2007.

24. Miller J. Tests reveal high chemical levels in kids' bodies. 2007.

25. National Safety Council. Health effects on children. 2004.

26. Acrylic W, Kong D. Study on the etiology and influencing factors of ADHD in children. Chin Matern Child Health Care. 2012;27 (34):5510-5512.

27. U.S. Environmental Protection Agency. Mercury. 2007.

28. Luo L, Liang Y, Yao Y. Study on the relationship between hyperactivity and autism in children and monoamine neurotransmitters in blood. Chin Clin Rehabil. 2002;6(19):2868. 
29. Zhang H, Yan Y. Molecular mechanism of neurotoxic effects of environmental neurotoxins (Manganese) on nerve cells. $J$ Env Occup Med. 2006;23(2):155-157.

30. Perera FP, Chang H, Tang D, Roen EL, Herbstman J, Margolis A. Early-life exposure to polycyclic aromatic hydrocarbons and ADHD behavior problems. PLoS One. 2014;9(11):1-10. doi:10.1371/journal. pone. 0111670

31. Huang D, Jin Y. Research progress on environmental risk factors of childhood autism. 2018 Academic Conference on Environment and Health-precise environmental health: a compilation of challenging papers for interdisciplinary collaboration. 2018.
32. Harris JB, Blain PG. Neurotoxicology: what the neurologist needs to know. J Neurol Neurosurg Psychiatry. 2004;75(suppl 3):iii29-iii34.

33. Guo D, Yang G. Environmental factors and autism. Prevent Control Chronic Dis China. 2012;20(5):599-603.

34. Dong D. The problem of "Problem Children" is lead poisoning. Chin Foreign Women's Health. 2012;1:26.

35. Liu Z. Research status of childhood autism. Prevent Control Chronic Dis China. 2008;16(1):104-107.

\section{Publish your work in this journal}

Psychology Research and Behavior Management is an international, peer-reviewed, open access journal focusing on the science of psychology and its application in behavior management to develop improved outcomes in the clinical, educational, sports and business arenas. Specific topics covered in the journal include: Neuroscience, memory and decision making; Behavior modification and management; Clinical applications; Business and sports performance management; Social and developmental studies; Animal studies. The manuscript management system is completely online and includes a very quick and fair peer-review system, which is all easy to use. Visit http://www. dovepress.com/testimonials.php to read real quotes from published authors. 